\title{
Wilke, Jürgen: 200 Jahre Karlsbader Beschlüsse. Zustandekommen, Inhalte, Folgen
}

\author{
Bremen: Edition Lumière 2019. 250 Seiten. Preis: $€ 29,80$
}

\author{
Daniel Gräßer
}

Angenommen: 9. Dezember 2020 / Online publiziert: 18. Dezember 2020

(C) Der/die Autor(en) 2020

Jürgen Wilke legt mit seinem Band zu den Karlsbader Beschlüssen eine erfreulich kurzweilige Aufarbeitung eben jener Ministerialkonferenz vor, die im böhmischen Kurort im Sommer 1819 stattfand. Erschienen bei Edition Lumière wirft er, äußerst passend, überfälliges Licht auf ein Ereignis historischer und nicht zuletzt kommunikationspolitischer Bedeutung, das dem Verfasser dieser Rezension zugegebenermaßen zuvor nur unzureichend geläufig war. Anlässlich des zweihundertjährigen Jubiläums der Beschlüsse einerseits, vor allem jedoch auch vor dem Hintergrund weltweit nicht nur wahrnehmbarer, sondern messbarer Bedrohung und Beschneidung von Presse- und Meinungsfreiheitsrechten, soll mit dem Band der Stellenwert des Kongresses und seiner Beschlüsse wieder im kollektiven Gedächtnis mindestens der Publizistik- und Kommunikationswissenschaft verankert werden.

Nach den napoleonischen Befreiungskriegen versammelten sich in Karlsbad Vertreter der deutschsprachigen Kaiser- und Königreiche sowie Fürstentümer des im Zuge des Wiener Kongresses 1814/15 neu gegründeten Deutschen Bundes, um den ersten zarten republikanischen Hoffnungen auf Presse- und Meinungsfreiheit, die in einzelnen Gliedstaaten durchaus galten, wieder den Garaus zu machen. Die getroffenen Einigungen warfen die medienpolitische Entwicklung auf dem Weg zu einer liberal-demokratischen, freiheitlichen Gesellschaft um viele Jahrzehnte zurück.

Auf der Basis eigener Quellenanalyse von historischen Zeitungsartikeln sowie historischer literarischer Publikationen arbeitet Jürgen Wilke die beteiligten Akteure, ihre Konstellationen zueinander, divergierende Positionen und vor allem Ergebnisse in chronologischer Reihenfolge ansprechend auf. Die älteste analysierte Quelle

D. Gräßer $(\bowtie)$

Institut für Publizistik- und Kommunikationswissenschaft AS

Kommunikationspolitik/Medienökonomie, Freie Universität Berlin, Garystr. 55, 14195 Berlin,

Deutschland

E-Mail: daniel.graesser@fu-berlin.de 
erschien noch vor dem eigentlichen Abschluss der Verhandlungen, andere im Nachgang der Konferenz, in der Zeit vor und nach der niedergeschlagenen März-Revolution, der Gründung des Deutschen Kaiserreichs, der Weimarer Republik bis hin zur besonders hervorgehobenen Dissertation Eberhard Büssems von 1974, die vielleicht gerade noch als zeitgenössisch bezeichnet werden kann. Das vorliegende Werk ist somit keinesfalls das erste, das die Karlsbader Beschlüsse aufarbeitet, sondern trägt vielmehr zur Vergegenwärtigung eines in Vergessenheit geratenen Ereignisses bei.

Klar nachvollziehbar und überzeugend gelingt Wilke die Beschreibung der Umstände und Geschehnisse, aufgrund derer die Konferenz in Karlsbad für nötig erachtet und mit denen ihre Beschlüsse letztlich legitimiert wurden. Neben der Schilderung historischer Ereignisse erreicht er dies unter anderem durch Wiedergabe der Briefwechsel des österreichischen Außenministers Metternich sowie seines Hofrates Gentz, die beide eine richtungsweisende Rolle bei der Wiedereinschränkung der vereinzelt im Deutschen Bund praktizierten Pressefreiheit einnahmen.

Für die Darstellung der einzelnen, im Rahmen der Karlsbader Konsultationen thematisierten Regulierungsbereiche, wie man sie heute wohl nennen könnte, greift Wilke auf die publizierte vollumfassende Sammlung der Konferenz-Dokumente von Klüber zurück. Erschienen ist sie im Jahre 1845, als die verlegerischen Einschränkungen noch Gesetzeskraft hatten. Durch ihre zumindest oberflächliche Neutralität fiel sie diesen jedoch nicht zum Opfer, handelte es sich doch schlicht um die schriftliche Wiedergabe des geltenden Rechts. Anhand der Dokumente zeichnet Jürgen Wilke die Argumentationslinien der Konferenzteilnehmer transparent nach, gespickt mit der einen oder anderen auch interpretativen Einordnung. Leider recht schmerzlich erinnert die Schilderung der jahrzehntelangen Verlängerung, letztlich Entfristung, der ursprünglich auf fünf Jahre begrenzten Pressegesetze, an die Marginalisierung parlamentarischer Diskussion und Kontrolle, die bis zur Entmachtung reichte, wie sie auch die erste Demokratie auf deutschem Boden ereilte, und höchst aktuell im Corona-Jahr in einzelnen Ländern der Europäischen Union durch Notstandsgesetzgebung geschieht.

Die Beschlüsse führten vereinzelt zu geradezu absurd komödienhaften Umständen, sei es die hohe Fluktuation auf den letztlich unbeliebten Zensoren-Positionen oder die von der Obrigkeit unerwünschten Textlücken in gedruckten Publikationen, wenn zensierte Beiträge nicht mehr rechtzeitig durch vorgehaltene Alternativbeiträge ersetzt werden konnten. Gesondert hervorgehoben werden soll an dieser Stelle unbedingt die umfangreiche Darlegung des Bespitzelungssystems, auch hier wieder vor allem wegen seiner bedauerlichen Nähe zu Überwachungssystemen in der jüngeren deutschen Vergangenheit. Die insgesamt hohe Informationsdichte des Buches kulminiert in diesen Kapiteln.

Die Schilderungen von Biografien und Beziehungen einzelner Hauptakteure zueinander, auch des historischen Ablaufs der Ereignisse, lässt dabei einen bald romanhaften Lesefluss mit durchaus spannenden Elementen entstehen - nicht unüblich in historischen Darstellungen, in jedem Fall ein Pluspunkt dieser insgesamt empfehlenswerten Publikation.

Der vorliegende Band ist somit insbesondere für ForscherInnen im Feld der Kommunikationsgeschichte interessant, aber durchaus auch für Governance- und StrukturforscherInnen, die aktuelle Diskurse im Wissen um Vorläufer und Parallelen aus 
der Vergangenheit ergründen möchten. Wünschenswert wäre selbstredend, wenn die zeitgemäße Aufarbeitung vor allem auch dazu führen würde, junge Studierende für das Feld zu begeistern und ihre Perspektive um mindestens zwei Jahrhunderte zu erweitern.

$\mathrm{Zu}$ guter Letzt weiß die Buchpublikation mit Kupferstichabbildungen vieler der beteiligten Akteure, den Titelseiten verschiedener zitierter Periodika der Zeit sowie weiterer größerer und kleinerer archivarischer Überraschungen (so zum Beispiel einer Fotografie der polizeilichen Meldeliste der Stadt Karlsbad vom Tag der Anreise Metternichs) auch ästhetisch zu gefallen.

Funding Open Access funding enabled and organized by Projekt DEAL.

Open Access Dieser Artikel wird unter der Creative Commons Namensnennung 4.0 International Lizenz veröffentlicht, welche die Nutzung, Vervielfältigung, Bearbeitung, Verbreitung und Wiedergabe in jeglichem Medium und Format erlaubt, sofern Sie den/die ursprünglichen Autor(en) und die Quelle ordnungsgemäß nennen, einen Link zur Creative Commons Lizenz beifügen und angeben, ob Änderungen vorgenommen wurden.

Die in diesem Artikel enthaltenen Bilder und sonstiges Drittmaterial unterliegen ebenfalls der genannten Creative Commons Lizenz, sofern sich aus der Abbildungslegende nichts anderes ergibt. Sofern das betreffende Material nicht unter der genannten Creative Commons Lizenz steht und die betreffende Handlung nicht nach gesetzlichen Vorschriften erlaubt ist, ist für die oben aufgeführten Weiterverwendungen des Materials die Einwilligung des jeweiligen Rechteinhabers einzuholen.

Weitere Details zur Lizenz entnehmen Sie bitte der Lizenzinformation auf http://creativecommons.org/ licenses/by/4.0/deed.de.

Daniel Gräßer ist wissenschaftlicher Mitarbeiter an der Arbeitsstelle Kommunikationspolitik/Medienökonomie am Institut für Publizistik- und Kommunikationswissenschaft der Freien Universität Berlin. 